\title{
SOBOLEV'S INEQUALITIES FOR HERZ-MORREY-ORLICZ SPACES ON THE HALF SPACE
}

\author{
Yoshihiro Mizuta, Takao OHno And Tetsu Shimomura
}

Abstract. We introduce Herz-Morrey-Orlicz spaces on the half space, and study the boundedness of the Hardy-Littlewood maximal operator. As an application, we establish Sobolev's inequality for Riesz potentials of functions in such spaces, which is one of mixed norm type inequalities. Mathematics subject classification (2010): 31B15, 46E35. inequality.

Keywords and phrases: Herz-Morrey-Orlicz spaces, maximal functions, Riesz potentials, Sobolev's

\section{REFERENCES}

[1] D. R. Adams, A note on Riesz potentials, Duke Math. J. 42 (1975), 765-778.

[2] D. R. Adams And L. I. Hedberg, Function spaces and potential theory, Springer, 1996.

[3] D. R. Adams And J. Xiao, Morrey spaces in harmonic analysis, Ark. Mat. 50, 2 (2012), 201-230.

[4] B. BoJARs Ki AND P. HaJŁasz, Pointwise inequalities for Sobolev functions and some applications, Studia Math. 106, 1 (1993), 77-92.

[5] V. I. Burenkov, A. Gogatishvili, V. S. Guliyev and R. Ch. Mustafayev, Boundedness of the fractional maximal operator in local Morrey-type spaces, Complex Var. Elliptic Equ. 55, 8-10 (2010), 739-758.

[6] V. I. Burenkov, A. Gogatishvili, V. S. Guliyev and R. Ch. Mustafayev, Boundedness of the Riesz potential in local Morrey-type spaces, Potential Anal. 35, 1 (2011), 67-87.

[7] F. Chiarenza And M. FrasCa, Morrey spaces and Hardy-Littlewood maximal function, Rend. Mat. 7 (1987), 273-279.

[8] Z. FU, Y. LIN AND S. LU, $\lambda$-central BMO estimates for commutators of singular integral operators with rough kernels, Acta Math. Sin. (Engl. Ser.) 24, 3 (2008), 373-386.

[9] J. L. LEWIS, On very weak solutions of certain elliptic systems, Comm. Partial Differential Equations 18 (9) (10) (1993), 1515-1537.

[10] X. Li AND D. YAng, Boundedness of some sublinear operators on Herz spaces, Illinois J. Math. 40 (1996), 484-501.

[11] F.-Y. Maeda, Y. Mizuta, T. Ohno and T. Shimomura, Boundedness of maximal operators and Sobolev's inequality on Musielak-Orlicz-Morrey spaces, Bull. Sci. math. 137 (2013), 76-96.

[12] Y. Mizuta AND T. OHNo, Boundedness of the maximal operator and Sobolev's inequality on nonhomogeneous central Herz-Morrey-Orlicz spaces, Nonlinear Anal. 128 (2015), 325-347.

[13] C. B. Morrey, On the solutions of quasi-linear elliptic partial differential equations, Trans. Amer. Math. Soc. 43 (1938), 126-166.

[14] E. NAKAI, Hardy-Littlewood maximal operator, singular integral operators and the Riesz potentials on generalized Morrey spaces, Math. Nachr. 166 (1994), 95-103.

[15] E. NAKAI, Calderón-Zygmund operators on Orlicz-Morrey spaces and modular inequalities, Banach and Function Spaces II, 393-410, Yokohama Publ., Yokohama, 2008.

[16] E. NAKAI, Orlicz-Morrey spaces and the Hardy-Littlewood maximal function, Studia Math. 188 (2008), 193-221.

[17] J. PeETRE, On the theory of $L_{p, \lambda}$ spaces, J. Funct. Anal. 4 (1969), 71-87.

[18] E. M. STEIN, Singular integrals and differentiability properties of functions, Princeton Univ. Press, Princeton, 1970. 